\title{
A NONCONVENTIONAL INVARIANCE PRINCIPLE FOR RANDOM FIELDS
}

\author{
YURI KIFER \\ INSTITUTE OF MATHEMATICS \\ THE HEBREW UNIVERSITY OF JERUSALEM
}

\begin{abstract}
In [17] we obtained a nonconventional invariance principle (functional central limit theorem) for sufficiently fast mixing stochastic processes with discrete and continuous time. In this paper we derive a nonconventional invariance principle for sufficiently well mixing random fields.
\end{abstract}

\section{INTRODUCTION}

Nonconventional ergodic theorems (see [12]) known also after 3 as polinomial ergodic theorems studied the limits of expressions having the form $1 / N \sum_{n=1}^{N} T^{q_{1}(n)} f_{1} \cdots T^{q_{\ell}(n)} f_{\ell}$ where $T$ is a weakly mixing measure preserving transformation, $f_{i}$ 's are bounded measurable functions and $q_{i}$ 's are polynomials taking on integer values on the integers. Originally, these results were motivated by applications to multiple recurrence for dynamical systems taking functions $f_{i}$ being indicators of some measurable sets. Later such results were extended to the case when $q_{i}$ 's are polinomials on $\mathbb{Z}^{\nu}$ (see [18) and to some $\mathbb{Z}^{\nu}$ actions (see [2]).

Using the language of probability this kind of results may be called nonconventional laws of large numbers and as a natural follow up we arrived at the invariance principle (functional central limit theorem) in [17 showing convergence in distribution to Gaussian processes for expressions of the form

$$
1 / \sqrt{N} \sum_{0 \leq n \leq N t}\left(F\left(X\left(q_{1}(n)\right), \ldots, X\left(q_{\ell}(n)\right)\right)-\bar{F}\right)
$$

where $X(n), n \geq 0$ is a sufficiently fast $\alpha, \rho$ or $\psi$-mixing vector valued process with some moment conditions and stationarity properties, $F$ is a continuous function with polinomial growth and certain regularity properties, $\bar{F}=\int F d(\mu \times \cdots \times \mu)$, $\mu$ is the distribution of each $X(n), q_{j}(n)=j n, j \leq k \leq \ell$ and $q_{j}, j=k+1, \ldots, \ell$ are positive functions taking on integer values on integers with some growth conditions which are satisfied, for instance, when $q_{i}$ 's are polynomials of growing degrees.

The goal of this paper is to prove an invariance principle type result when $n \in \mathbb{Z}^{\nu}$ is multidimensional. This can be done either by considering functions $q_{i}: \mathbb{Z}^{\nu} \rightarrow \mathbb{Z}_{+}$ with $X(n), n \geq 0$ being again a vector valued stochastic process or, more generally, considering maps $q_{i}: \mathbb{Z}^{\nu} \rightarrow \mathbb{Z}^{\nu}$ taking now $X(n), n \in \mathbb{Z}^{\nu}$ to be a vector valued

Date: November 11, 2018.

2000 Mathematics Subject Classification. Primary: 60F17 Secondary: 60G60.

Key words and phrases. random fields, limit theorems, mixing. 
random field which will be our setup in this paper. Namely, for $t=\left(t_{1}, \ldots, t_{\nu}\right) \in$ $[0,1]^{\nu}$ and a positive integer $N$ we consider expressions of the form

$$
\xi_{N}(t)=N^{-\nu / 2} \sum_{n=\left(n_{1}, \ldots, n_{\nu}\right): 0 \leq n_{i} \leq N t_{i} \forall i}\left(F\left(X\left(q_{1}(n)\right), \ldots, X\left(q_{\ell}(n)\right)\right)-\bar{F}\right)
$$

where $X(n), n \in \mathbb{Z}^{\nu}$ is a sufficiently well mixing vector valued random field, with some moment conditions and stationarity properties, $F$ and $\bar{F}$ are similar to above, $q_{j}(n)=j n, j \leq k$ and $q_{i}: \mathbb{Z}^{\nu} \rightarrow \mathbb{Z}^{\nu}, i=k+1, \ldots, \ell \operatorname{map} \mathbb{Z}_{+}^{\nu}=\left\{n=\left(n_{1}, \ldots, n_{\nu}\right) \in\right.$ $\left.\mathbb{Z}^{\nu}: n_{i} \geq 0, i=1, \ldots, \nu\right\}$ into itself. Assuming some growth conditions of $\left|q_{i}\right|, i>k$ in $|n|$ we will show that the random field $\xi_{N}(t)$ converges in distribution to a Gaussian random field on $[0,1]^{\nu}$.

In [17] we were able to obtain the latter result for one dimensional $n$ relying on martingale approximations and martingale limit theorems but for random fields this machinery is not readily available. Still, we are able to combine some of mixingale technique from [19 and 20 together with an appropriate grouping of summands in (1.2) in order to obtain both convergence of finite dimensional distributions and the tightness of infinite dimensional ones. Other known methods which work successfully when proving limit theorems for random fields (see, for instance, 5, 7, [8] and 21] ) rely one way or another on characteristic functions (or other devices based on weak dependence) which are hard to deal with in the nonconventional setup as demonstrated in [15] in view of the strong dependence of the summands in (1.2) on the far away members of the random field. For specific lattice models with sufficiently good mixing properties to fit our setup we refer the reader to [1] and references there.

\section{Preliminaries AND main Results}

Our setup consists of a $\wp$-dimensional vector random field $\{X(n), n \in$ $\left.\mathbb{Z}^{\nu}, X(n) \in \mathbb{R}^{\wp}\right\}$ on a probability space $(\Omega, \mathcal{F}, P)$ and of a family of $\sigma$-algebras $\mathcal{F}_{\Gamma} \subset \mathcal{F}, \Gamma \subset \mathbb{Z}^{\nu}$ such that $\mathcal{F}_{\Gamma} \subset \mathcal{F}_{\Delta}$ if $\Gamma \subset \Delta \subset \mathbb{Z}^{\nu}$. It is often convenient to measure the dependence between two sub $\sigma$-algebras $\mathcal{G}, \mathcal{H} \subset \mathcal{F}$ via the quantities

$$
\varpi_{q, p}(\mathcal{G}, \mathcal{H})=\sup \left\{\|E(g \mid \mathcal{G})-E g\|_{p}: g \text { is } \mathcal{H}-\text { measurable and }\|g\|_{q} \leq 1\right\}
$$

where the supremum is taken over real functions and $\|\cdot\|_{r}$ is the $L^{r}(\Omega, \mathcal{F}, P)$-norm. Then more familiar $\alpha, \rho, \phi$ and $\psi$-mixing (dependence) coefficients can be expressed via the formulas (see [], Ch. 4 ),

$$
\begin{gathered}
\alpha(\mathcal{G}, \mathcal{H})=\frac{1}{4} \varpi_{\infty, 1}(\mathcal{G}, \mathcal{H}), \rho(\mathcal{G}, \mathcal{H})=\varpi_{2,2}(\mathcal{G}, \mathcal{H}) \\
\phi(\mathcal{G}, \mathcal{H})=\frac{1}{2} \varpi_{\infty, \infty}(\mathcal{G}, \mathcal{H}) \text { and } \psi(\mathcal{G}, \mathcal{H})=\varpi_{1, \infty}(\mathcal{G}, \mathcal{H}) .
\end{gathered}
$$

We set also

$$
\varpi_{q, p}(r)=\sup _{\Gamma, \Delta: \operatorname{dist}(\Gamma, \Delta) \geq r}\left(|\Gamma \cup \Delta|^{-1} \varpi_{q, p}\left(\mathcal{F}_{\Gamma}, \mathcal{F}_{\Delta}\right)\right)
$$

where $\Gamma$ and $\Delta$ are finite nonempty subsets of $\mathbb{Z}^{\nu}, \operatorname{dist}(\Gamma, \Delta)=\inf _{n \in \Gamma, \tilde{n} \in \Delta}|n-\tilde{n}|$ and we write $|\Gamma|$ for cardinality of a set $\Gamma$ while, as usual, for numbers or vectors $|\cdot|$ will denote their absolute values or lengths. As shown in [10] imposing decay conditions on dependence coefficients which do not take into account sizes of sets $\Gamma$ and $\Delta$ as in (2.2) would exclude from our setup simple examples of Gibbs random 
fields. Define also

$$
\alpha(l)=\frac{1}{4} \varpi_{\infty, 1}(l), \rho(l)=\varpi_{2,2}(l), \phi(l)=\varpi_{\infty, \infty}(l) \text { and } \psi(l)=\varpi_{1, \infty}(l) .
$$

Our setup includes also conditions on the approximation rate

$$
\beta_{p}(r)=\sup _{n \in \mathbb{Z}^{\nu}}\left\|X(n)-E\left(X(n) \mid \mathcal{F}_{U_{r}(n)}\right)\right\|_{p}
$$

where $U_{r}(n)=\left\{\tilde{n} \in \mathbb{Z}^{\nu}:|n-\tilde{n}| \leq r\right\}$ is the $r$-neghborhood on $n$ in $\mathbb{Z}^{\nu}$. Furthermore, we do not require stationarity of the random field $X(n), n \in \mathbb{Z}^{\nu}$ assuming only that the distribution of $X(n)$ does not depend on $n$ and the joint distribution of $\left\{X(n), X\left(n^{\prime}\right)\right\}$ depends only on $n-n^{\prime}$ which we write for further references by

$$
X(n) \stackrel{d}{\sim} \mu \text { and }\left(X(n), X\left(n^{\prime}\right)\right) \stackrel{d}{\sim} \mu_{n-n^{\prime}} \text { for all } n, n^{\prime}
$$

where $Y \stackrel{d}{\sim} Z$ means that $Y$ and $Z$ have the same distribution.

Next, let $F=F\left(x_{1}, \ldots, x_{\ell}\right), x_{j} \in \mathbb{R}^{\wp}$ be a function on $\mathbb{R}^{\wp \ell}$ such that for some $\iota, K>0, \kappa \in(0,1]$ and all $x_{i}, y_{i} \in \mathbb{R}^{\wp}, i=1, \ldots, \ell$, we have

$$
\left|F\left(x_{1}, \ldots, x_{\ell}\right)-F\left(y_{1}, \ldots, y_{\ell}\right)\right| \leq K\left(1+\sum_{j=1}^{\ell}\left|x_{j}\right|^{\iota}+\sum_{j=1}^{\ell}\left|y_{j}\right|^{\iota}\right) \sum_{j=1}^{\ell}\left|x_{j}-y_{j}\right|^{\kappa}
$$

and

$$
\left|F\left(x_{1}, \ldots, x_{\ell}\right)\right| \leq K\left(1+\sum_{j=1}^{\ell}\left|x_{j}\right|^{\iota}\right) .
$$

Our assumptions on $F$ enable us to include, for instance, products $F\left(x_{1}, \ldots, x_{\ell}\right)=$ $x_{11} x_{22} \cdots x_{\ell \ell}$, where $x_{i}=\left(x_{i 1}, \ldots, x_{i \ell}\right) \in \mathbb{R}^{\ell}$, which is sometimes useful. To simplify formulas we assume a centering condition

$$
\bar{F}=\int F\left(x_{1}, \ldots, x_{\ell}\right) d \mu\left(x_{1}\right) \cdots d \mu\left(x_{\ell}\right)=0
$$

which is not really a restriction since we always can replace $F$ by $F-\bar{F}$.

Our goal is to prove an invariance principle (functional central limit theorem) for $\xi_{N}(t), t \in[0,1]^{\nu}$ defined by (1.2) where $q_{j}(n)=j n$ if $j=1,2, \ldots, k \leq \ell$ for some given positive integers $k, \ell$ and if $k<\ell$ then $q_{j}: \mathbb{Z}^{\nu} \rightarrow \mathbb{Z}^{\nu}, j=k+1, \ldots, \ell$ satisfy the conditions below. Before we formulate them observe that already the case $k=\ell$ is of major interest and we add $q_{j}^{\prime} s$ with $j>k$ which grow faster than linearly mostly for the sake of completeness which under appropriate assumptions does not cause substantial problems. We assume that $\left|q_{1}(n)\right|<\left|q_{2}(n)\right|<\cdots<\left|q_{\ell}(n)\right|$ whenever $|n| \neq 0$ and $n \in \mathbb{Z}_{+}^{\nu}=\left\{m=\left(m_{1}, \ldots, m_{\nu}\right) \in \mathbb{Z}^{\nu}: m_{i} \geq 0 \forall i\right\}$. Furthermore, we assume that for $k+1 \leq i \leq \ell$,

$$
\begin{gathered}
\inf _{|\tilde{n}|>|n|}\left(\left|q_{i}(\tilde{n})\right|-\left|q_{i}(n)\right|\right)(|\tilde{n}|-|n|)^{-1}>0, \\
\lim _{|n| \rightarrow \infty} \inf _{\tilde{n}: \tilde{n} \neq n}\left(\left|q_{i}(\tilde{n})-q_{i}(n)\right|-|\tilde{n}-n|\right)=\infty, \\
q_{i}(n) \neq q_{i}(\tilde{n}) \text { if } n \neq \tilde{n}, \lim _{|n| \rightarrow \infty} \min _{l<i}\left(\left|q_{i}(n)\right|-\left|q_{l}(n)\right|-|n|\right)=\infty
\end{gathered}
$$

and for any $\varepsilon>0$,

$$
\lim _{|n| \rightarrow \infty \tilde{n}:|\tilde{n}| \geq \varepsilon|n|} \min _{l<i}\left(\left|q_{i}(\tilde{n})\right|-\left|q_{l}(n)\right|-|\tilde{n}-n|\right)=\infty .
$$


For each $\theta>0$ set

$$
\gamma_{\theta}^{\theta}=\|X\|_{\theta}^{\theta}=E|X(n)|^{\theta}=\int|x|^{\theta} d \mu .
$$

Our main result relies on

2.1. Assumption. With $d=(\ell-1) \wp$ there exist $p, q \geq 1, m \geq 4$ and $\delta>0$ with $\delta \leq \kappa, p \kappa>d$ satisfying

$$
\begin{gathered}
\sum_{l=0}^{\infty} l^{5 \nu} \varpi_{q, p}(l)=\theta(p, q)<\infty, \\
\sum_{r=0}^{\infty} r^{5 \nu} \beta_{q}^{\delta}(r)<\infty, \\
\gamma_{m}<\infty, \gamma_{2 q \iota}<\infty \text { with } \frac{1}{2} \geq \frac{1}{p}+\frac{\iota+1}{m}+\frac{\delta}{q} .
\end{gathered}
$$

In order to give a detailed statement of our main result as well as for its proof it will be essential to represent the function $F=F\left(x_{1}, x_{2}, \ldots, x_{\ell}\right)$ in the form

$$
F=F_{1}\left(x_{1}\right)+\cdots+F_{\ell}\left(x_{1}, x_{2}, \ldots, x_{\ell}\right)
$$

where for $i<\ell$,

$$
\begin{gathered}
F_{i}\left(x_{1}, \ldots, x_{i}\right)=\int F\left(x_{1}, x_{2}, \ldots, x_{\ell}\right) d \mu\left(x_{i+1}\right) \cdots d \mu\left(x_{\ell}\right) \\
-\int F\left(x_{1}, x_{2}, \ldots, x_{\ell}\right) d \mu\left(x_{i}\right) \cdots d \mu\left(x_{\ell}\right)
\end{gathered}
$$

and

$$
F_{\ell}\left(x_{1}, x_{2}, \ldots, x_{\ell}\right)=F\left(x_{1}, x_{2}, \ldots, x_{\ell}\right)-\int F\left(x_{1}, x_{2}, \ldots, x_{\ell}\right) d \mu\left(x_{\ell}\right)
$$

which ensures, in particular, that

$$
\int F_{i}\left(x_{1}, x_{2}, \ldots, x_{i-1}, x_{i}\right) d \mu\left(x_{i}\right) \equiv 0 \quad \forall \quad x_{1}, x_{2}, \ldots, x_{i-1} .
$$

We write $t=\left(t_{1}, \ldots, t_{\nu}\right) \geq s=\left(s_{1}, \ldots, s_{\nu}\right)$ if $t_{i} \geq s_{i}$ for all $i$ and for such $s, t \in[0,1]^{\nu}$ we set $\Delta_{N}(s, t)=\left\{n=\left(n_{1}, \ldots, n_{\nu}\right) \in \mathbb{Z}^{\nu}: N s_{i} \leq n_{i} \leq N t_{i} \forall i\right\}$ and $\Delta_{N}(t)=$ $\Delta_{N}(0, t)$. These together with (2.16) $-(2.18)$ enable us to represent $\xi_{N}(t)$ given by (1.2) in the form

$$
\xi_{N}(t)=\sum_{i=1}^{k} \xi_{i, N}(i t)+\sum_{i=k+1}^{\ell} \xi_{i, N}(t)
$$

where for $1 \leq i \leq k$,

$$
\xi_{i, N}(t)=N^{-\nu / 2} \sum_{n \in \Delta_{N}(t / i)} F_{i}(X(n), X(2 n), \ldots, X(i n))
$$

and for $i \geq k+1$,

$$
\xi_{i, N}(t)=N^{-\nu / 2} \sum_{n \in \Delta_{N}(t)} F_{i}\left(X\left(q_{1}(n)\right), \ldots, X\left(q_{i}(n)\right)\right) .
$$

Next, we define a matrix $\left(D_{i, j}, 1 \leq i, j \leq k\right)$ which appears in the limiting covariances formula in our main result below. For any $i, j \leq k$ set

$$
D_{i, j}=\frac{v}{i j} \sum_{u \in \mathbb{Z}^{\nu}} c_{i, j}(u)
$$


where $v$ is the greatest common divisor of $i$ and $j, c_{i, j}(u)=0$ if $v$ does not divide all components of $u \in \mathbb{Z}^{\nu}$ and for $i^{\prime}=i / v, j^{\prime}=j / v$,

$$
\begin{gathered}
c_{i, j}(v u)=\int F_{i}\left(x_{1}, \ldots, x_{i}\right) F_{j}\left(y_{1}, \ldots, y_{j}\right) \prod_{\alpha=1}^{v} d \mu_{\alpha u}\left(x_{\alpha i^{\prime}}, y_{\alpha j^{\prime}}\right) \\
\prod_{\sigma \notin\left\{i^{\prime}, 2 i^{\prime}, \ldots, v i^{\prime}\right\}} d \mu\left(x_{\sigma}\right) \prod_{\sigma^{\prime} \notin\left\{j^{\prime}, 2 j^{\prime}, \ldots, v j^{\prime}\right\}} d \mu\left(y_{\sigma^{\prime}}\right)
\end{gathered}
$$

with $\mu_{0}$ being the diagonal measure, i.e. $\int f(x, y) d \mu_{0}(x, y)=\int f(x, x) d \mu(x)$.

2.2. Theorem. Suppose that the conditions 2.4)-2.11) and Assumption 2.1 hold true then each random field $\xi_{i, N}(t), i=1,2, \ldots, \ell$ converges in distribution as $N \rightarrow \infty$ to a Gaussian random field $\eta_{i}(t)$. Moreover, $\left(\eta_{1}(t), \eta_{2}(t), \ldots, \eta_{\ell}(t)\right)$ is an $\ell$-dimensional Gaussian random field such that $\eta_{i}(t), i \leq k$ have covariances

$$
E \eta_{i}(s) \eta_{j}(t)=D_{i, j} \prod_{l=1}^{\nu} \min \left(s_{l}, t_{l}\right), i, j \leq k
$$

with matrix $D_{i, j}$ defined by (2.22) while the random fields $\eta_{i}(t), i \geq k+1$ are independent of each other and of $\eta_{j}$ 's with $j \leq k$ and have variances

$$
E\left|\eta_{i}(t)\right|^{2}=\int\left|F_{i}\left(x_{1}, x_{2}, \ldots, x_{i}\right)\right|^{2} d \mu\left(x_{1}\right) d \mu\left(x_{2}\right) \cdots d \mu\left(x_{i}\right), i \geq k+1 .
$$

Finally, $\xi_{N}(t)$ converges in distribution to a Gaussian random field $\xi(t)$ which can be represented in the form

$$
\xi(t)=\sum_{i=1}^{k} \eta_{i}(i t)+\sum_{i=k+1}^{\ell} \eta_{i}(t) .
$$

In order to understand our assumptions observe that $\varpi_{q, p}$ is clearly nonincreasing in $q$ and non-decreasing in $p$. Hence, for any pair $p, q \geq 1$,

$$
\varpi_{q, p}(n) \leq \psi(n)
$$

Furthermore, by the real version of the Riesz-Thorin interpolation theorem or the Riesz convexity theorem (see [13, Section 9.3 and [11, Section VI.10.11) whenever $\theta \in[0,1], 1 \leq p_{0}, p_{1}, q_{0}, q_{1} \leq \infty$ and

$$
\frac{1}{p}=\frac{1-\theta}{p_{0}}+\frac{\theta}{p_{1}}, \frac{1}{q}=\frac{1-\theta}{q_{0}}+\frac{\theta}{q_{1}}
$$

then

$$
\varpi_{q, p}(n) \leq 2\left(\varpi_{q_{0}, p_{0}}(n)\right)^{1-\theta}\left(\varpi_{q_{1}, p_{1}}(n)\right)^{\theta} .
$$

In particular, using the obvious bound $\varpi_{q_{1}, p_{1}} \leq 2$ valid for any $q_{1} \geq p_{1}$ we obtain from (2.25) for pairs $(\infty, 1),(2,2)$ and $(\infty, \infty)$ that for all $q \geq p \geq 1$,

$$
\begin{gathered}
\varpi_{q, p}(n) \leq(2 \alpha(n))^{\frac{1}{p}-\frac{1}{q}}, \varpi_{q, p}(n) \leq 2^{1+\frac{1}{p}-\frac{1}{q}}(\rho(n))^{1-\frac{1}{p}+\frac{1}{q}} \\
\text { and } \varpi_{q, p}(n) \leq 2^{1+\frac{1}{p}}(\phi(n))^{1-\frac{1}{p}} .
\end{gathered}
$$

We observe also that by the Hölder inequality for $q \geq p \geq 1$ and $\alpha \in(0, p / q)$,

$$
\beta_{q}(r) \leq 2^{1-\alpha}\left[\beta_{p}(r)\right]^{\alpha} \gamma_{\frac{p q(1-\alpha)}{p-q \alpha}}^{1-\alpha}
$$

with $\gamma_{\theta}$ defined in (2.12). Thus, we can formulate Assumption 2.1] in terms of more familiar $\alpha, \rho, \phi$, and $\psi$-mixing coefficients and with various moment conditions. It follows also from (2.25) that if $\varpi_{q, p}(n) \rightarrow 0$ as $n \rightarrow \infty$ for some $q \geq p \geq 1$ then

$$
\varpi_{q, p}(n) \rightarrow 0 \text { as } n \rightarrow \infty \text { for all } q>p \geq 1,
$$


and so (2.28) holds true under Assumption 2.1

In order to prove Theorem 2.2 we will represent $\xi_{i, N}(t)$ in the form $\sum_{1 \leq l<N} Z_{t, N}(l)$ where now $l$ is one dimensional which together with estimates of the next section will enable us to apply central limit theorems for mixingale arrays (see [19] and [20]). This will lead to Gaussian one dimensional distributions in the limit but combining this with a kind of the Cramér-Wold argument, covariances computation in Section 4 and tightness estimates of Section 5 will yield appropriate Gaussian random fields as asserted in the theorem. Recall (see [17), that already in the one parameter case $\nu=1$ the process $\xi(t)$, in general, does not have independent increments so also in the random field case $\xi(t)$, in general, is not a multiparameter Brownian motion.

2.3. Remark. As a part of tightness estimates of Section [5 we will see that $\sup _{N \geq 1, t \in[0,1]^{\nu}} E\left|\xi_{i, N}(t)\right|^{4} \leq C<\infty$. Hence, applying the Borel-Cantelli lemma we obtain as a byproduct that if $S_{i, N}=N^{\nu / 2} \xi_{i, N}(t)$ and $S_{N}=N^{\nu / 2} \sum_{i=1}^{\ell} \xi_{i, N}(t)$ then with probability one

$$
\lim _{N \rightarrow \infty} \frac{1}{N^{\nu}} S_{i, N}(t)=0 \text { for each } i \text {, and so } \lim _{N \rightarrow \infty} \frac{1}{N^{\nu}} S_{N}(t)=0 .
$$

Still, we observe that this strong law of large numbers can be obtained under more general circumstances here since, in particular, we do not need for it convergence of covariances derived in Section 4 which requires, for instance, more specific assumptions on $q_{j}$ 's.

\section{BlOCKS AND MIXINGALE TYPE ESTIMATES}

We rely on the following result which appears as Corollary 3.6 in [17].

3.1. Proposition. Let $\mathcal{G}$ and $\mathcal{H}$ be $\sigma$-subalgebras on a probability space $(\Omega, \mathcal{F}, P)$, $X$ and $Y$ be d-dimensional random vectors and $f=f(x, \omega), x \in \mathbb{R}^{d}$ be a collection of random variables measurable with respect to $\mathcal{H}$ and satisfying

$$
\|f(x, \omega)-f(y, \omega)\|_{q} \leq C\left(1+|x|^{\iota}+|y|^{\iota}\right)|x-y|^{\kappa} \text { and }\|f(x, \omega)\|_{q} \leq C\left(1+|x|^{\iota}\right)
$$

where $q \geq 1$. Set $g(x)=E f(x, \omega)$. Then

$$
\|E(f(X, \cdot) \mid \mathcal{G})-g(X)\|_{v} \leq c\left(1+\|X\|_{b(\iota+2)}^{\iota+2}\right)\left(\varpi_{q, p}(\mathcal{G}, \mathcal{H})+\|X-E(X \mid \mathcal{G})\|_{q}^{\delta}\right)
$$

provided $\kappa-\frac{d}{p}>0, \frac{1}{v} \geq \frac{1}{p}+\frac{1}{b}+\frac{\delta}{q}$ with $c=c(C, \iota, \kappa, \delta, p, q, v, d)>0$ depending only on parameters in brackets. Moreover, let $x=(w, z)$ and $X=(W, Z)$, where $W$ and $Z$ are $d_{1}$ and $d-d_{1}$-dimensional random vectors, respectively, and let $f(x, \omega)=$ $f(w, z, \omega)$ satisfy (3.1) in $x=(w, z)$. Set $\tilde{g}(w)=E f(w, Z(\omega), \omega)$. Then

$$
\begin{gathered}
\|E(f(W, Z, \cdot) \mid \mathcal{G})-\tilde{g}(W)\|_{v} \leq c\left(1+\|X\|_{b(\iota+2)}^{\iota+2}\right) \\
\times\left(\varpi_{q, p}(\mathcal{G}, \mathcal{H})+\|W-E(W \mid \mathcal{G})\|_{q}^{\delta}+\|Z-E(Z \mid \mathcal{H})\|_{q}^{\delta}\right) .
\end{gathered}
$$

We will use the following notations

$$
\begin{gathered}
(3.4) F_{i, n, r}=F_{i, n, r}\left(x_{1}, x_{2}, \ldots, x_{i-1}, \omega\right)=E\left(F_{i}\left(x_{1}, x_{2}, \ldots, x_{i-1}, X(n)\right) \mid \mathcal{F}_{U_{r}(n)}\right), \\
Y_{i}\left(q_{i}(n)\right)=F_{i}\left(X\left(q_{1}(n)\right), \ldots, X\left(q_{i}(n)\right)\right) \text { and } Y_{i}(m)=0 \text { if } m \neq q_{i}(n) \text { for any } n, \\
X_{r}(n)=E\left(X(n) \mid \mathcal{F}_{U_{r}(n)}\right), Y_{i, r}\left(q_{i}(n)\right)=F_{i, q_{i}(n), r}\left(X_{r}\left(q_{1}(n)\right),\right. \\
\left.\ldots, X_{r}\left(q_{i-1}(n)\right), \omega\right) \text { and } Y_{i, r}(m)=0 \text { if } m \neq q_{i}(n) \text { for any } n ; \\
\bar{Y}_{i}(n)=Y_{i}(n)-E Y_{i}(n), \bar{Y}_{i, r}(n)=Y_{i, r}(n)-E Y_{i, r}(n) .
\end{gathered}
$$


For each $l \in \mathbb{Z}_{+}$introduce cubes $\square(l)=\left\{n=\left(n_{1}, \ldots, n_{\nu}\right) \in \mathbb{Z}^{\nu}: 0 \leq n_{i} \leq\right.$ $l$ for $i=1, \ldots, \nu\}$ and for $l<\tilde{l}$ we set also $\Upsilon(l, \tilde{l})=\square(\tilde{l}) \backslash \square(l)$. Fix some positive numbers $\frac{5}{11}(\tau+1)<3 \eta<\tau<1$ and set $a(1)=0, b(1)=1$ and for $j>1$,

$$
a(j)=b(j-1)+\left[(j-1)^{2 \eta}\right], b(j)=a(j)+\left[j^{\tau}\right] \text { and } r(j)=\left[j^{\eta}\right] .
$$

Set $\Delta_{N}^{(i)}(t)=\Delta_{N}(t / i)$ if $i \leq k$ and $\Delta_{N}^{(i)}(t)=\Delta_{N}(t)$ if $i \geq k+1$. We define now

$$
\begin{gathered}
V_{i, t, N}(l)=\sum_{n \in \Delta_{N}^{(i)}(t) \cap \Upsilon(a(l), b(l))} Y_{i, r(l)}\left(q_{i}(n)\right) \\
\text { and } W_{i, t, N}(l)=\sum_{n \in \Delta_{N}^{(i)}(t) \cap \Upsilon(b(l), a(l+1))} Y_{i, r(l)}\left(q_{i}(n)\right)
\end{gathered}
$$

The sets $\Upsilon(b(l), a(l+1))$ will play the role of gaps between $\Upsilon(a(l), b(l))$ and $\Upsilon(a(l+$ $1), b(l+1))$ and we will see that the random variables $W_{i, t, N}(l)$ can be disregarded for our purposes while dealing with the random variables $V_{i, t, N}(l)$ we will take advantage of our mixing conditions in order to show that their centered versions $\bar{V}_{i, t, N}(l)=V_{i, t, N}(l)-E V_{i, t, N}(l)$ satify mixingale estimates (see [19] and [20]) with respect to the nested family of $\sigma$-algebras $\mathcal{G}_{l}^{(i)}=\mathcal{F}_{\Gamma_{i}(l)}, l=0,1,2, \ldots$ where

$$
\Gamma_{i}(l)=\left\{n \in \mathbb{Z}_{+}^{\nu}: \operatorname{dist}\left(n, \cup_{j \leq i} q_{j}(\square(b(l)))\right) \leq r(l)\right\}
$$

and we take $\mathcal{G}_{l}^{(i)}$ to be the trivial $\sigma$-algebra $\{\emptyset, \Omega\}$ for $l<0$.

Namely, for any $u \in \mathbb{N}$ we have

$$
\begin{gathered}
\left\|E\left(\bar{V}_{i, t, N}(l) \mid \mathcal{G}_{l-u}^{(i)}\right)\right\|_{2} \leq \sum_{n \in \Delta_{N}(t) \cap \Upsilon(a(l), b(l))}\left\|E\left(\bar{Y}_{i, r(l)}\left(q_{i}(n)\right) \mid \mathcal{G}_{l-u}^{(i)}\right)\right\|_{2} \\
\leq|\Upsilon(a(l), b(l))| \max _{n \in \Upsilon(a(l), b(l))}\left\|E\left(\bar{Y}_{i, r(l)}\left(q_{i}(n)\right) \mid \mathcal{G}_{l-u}^{(i)}\right)\right\|_{2}
\end{gathered}
$$

where $|A|$ for a set $A$ denotes its cardinality. Next, for $u>l$,

$$
E\left(\bar{Y}_{i, r(l)}\left(q_{i}(n)\right) \mid \mathcal{G}_{l-u}^{(i)}\right)=0
$$

while for all $u \geq 2$ by the Cauchy inequality and the contraction property of conditional expectations

$$
\left\|E\left(\bar{Y}_{i, r(l)}\left(q_{i}(n)\right) \mid \mathcal{G}_{l-u}^{(i)}\right)\right\|_{2} \leq 2\left\|E\left(Y_{i, r(l)}\left(q_{i}(n)\right) \mid \mathcal{G}_{l-2}^{(i)}\right)\right\|_{2} .
$$

Observe that if $n \in \Upsilon(a(l), b(l))$ then $X=\left(X_{r(l)}\left(q_{1}(n)\right), \ldots, X_{r(l)}\left(q_{i-1}(n)\right)\right)$ is $\mathcal{G}_{l}^{(i-1)}$-measurable and for large $l$ we obtain also by the definition of $q_{i}$ for $i \leq k$ and by (2.8) and (2.11) for $i>k$ that

$$
\operatorname{dist}\left(\Gamma_{i}(l-2) \cup \Gamma_{i-1}(l), q_{i}(n)\right) \geq(l-1)^{\tau}
$$

taking into account that $a(l)>\frac{1}{1+\tau}(l-1)^{1+\tau}-(l-1)$. We can write also that

$$
b(l) \leq \frac{2}{1+\tau}(l+1)^{1+\tau},|\square(l)|=(l+1)^{\nu} \text { and }|\Upsilon(\tilde{l}, l)| \leq \nu(l-\tilde{l})(l+1)^{\nu-1} \text { if } l>\tilde{l} .
$$

Thus, applying Proposition 3.1 to the right hand side of (3.9) with $\mathcal{G}=$ $\mathcal{F}_{\Gamma_{i}(l-2) \cup \Gamma_{i-1}(l)}$ and $\mathcal{H}=\mathcal{F}_{U_{r(l)}\left(q_{i}(n)\right)}$ together with (3.10), (3.11), Assumption 2.1 and the contraction property of conditional expectations we obtain that

$$
\begin{gathered}
\left\|E\left(Y_{i, r(l)}\left(q_{i}(n)\right) \mid \mathcal{G}_{l-2}^{(i)}\right)\right\|_{2} \leq\left\|E\left(Y_{i, r(l)}\left(q_{i}(n)\right) \mid \mathcal{G}\right)\right\|_{2} \\
\leq C \varpi_{p, q}(\mathcal{G}, \mathcal{H}) \leq \tilde{C} l^{\nu(\tau+\eta+1)} \varpi_{p, q}\left((l-1)^{\tau}-l^{\eta}\right)
\end{gathered}
$$


for some $C, \tilde{C}>0$ independent of $n, l$ provided $p, q$ and $\delta$ satisfy the conditions of Assumption 2.1. Collecting (3.6)-(3.9), (3.11) and (3.12) we obtain that for any $u \geq 2$,

$$
\left\|E\left(\bar{V}_{i, t, N}(l) \mid \mathcal{G}_{l-u}^{(i)}\right)\right\|_{2} \leq \tilde{C} 2^{\nu(2+\tau)} l^{\nu(2 \tau+\eta+2)-1} \varpi_{p, q}\left((l-1)^{\tau}-l^{\eta}\right) .
$$

In order to incorporate (3.13) into the setup of mixingale arrays from [20] we consider the triangular array $\hat{V}_{i, t, N}(l)=N^{-\nu / 2} \bar{V}_{i, t, N}(l), l=1,2, \ldots, L(N) ; N=$ $1,2, \ldots$ where $L(N)=\min \{j: a(j+1) \geq N\}$. Observe that

$$
N \geq \sum_{1 \leq j \leq L(N)-1}\left[j^{\tau}\right] \geq(1+\tau)^{-1}(L(N)-1)^{1+\tau},
$$

and so

$$
L(N) \leq(N(1+\tau))^{1 /(1+\tau)}+1 \leq 2 N^{1 /(1+\tau)}+1 .
$$

Employing Lemma 4.2 from the next section together with (2.15), (3.11) and (3.14) we obtain that

$$
\left\|V_{i, t, N}(l)\right\|_{2}^{2} \leq C|\Upsilon(a(l), b(l))| \leq \frac{2 C \nu}{1+\tau} l^{\tau}(l+1)^{(1+\tau)(\nu-1)} \leq C_{1} N^{\nu-\frac{1}{1+\tau}}
$$

for some $C, C_{1}>0$ independent of $l \leq L(N), N, i$ and $t$. Observe that by the choice of $\tau$ and $\eta$ we have that $\nu(2 \tau+\eta+2)-1-5 \nu \tau<-1$ which together with (2.13) and (3.13) enables us to write

$$
\left\|E\left(\bar{V}_{i, t, N}(l) \mid \mathcal{G}_{l-u}^{(i)}\right)\right\|_{2} \leq C_{2} l^{-1} \leq C_{2} u^{-1}
$$

for some $C_{2} \geq 1$ independent of $l, N, i, t$ and $u=2,3, \ldots, l$. Set $\psi_{j}=$ $C_{2}(\max (1, j))^{-1}$ for $j=0,1,2, \ldots$ and $\sigma_{l, N}=C_{1} N^{-\frac{1}{2(1+\tau)}}$ for $l=1,2, \ldots, L(N)$. Then (3.8), (3.15) and (3.16) yield the first standard mixingale estimate

$$
\left\|E\left(\hat{V}_{i, t, N}(l) \mid \mathcal{G}_{l-u}^{(i)}\right)\right\|_{2} \leq \sigma_{l, N} \psi_{u}
$$

for all $u=0,1,2, \ldots$ and the conditions imposed on $\psi_{j}$ 's and $\sigma_{l, N}$ 's in 20. can be easily verified, as well. The second standard mixingale estimate (see [20]) is trivial in our case since $\bar{V}_{i, t, N}(l)$ is $\mathcal{G}_{l+u}^{(i)}$-measurable for any $u \geq 1$, and so

$$
E\left(\bar{V}_{i, t, N}(l) \mid \mathcal{G}_{l+u}^{(i)}\right)-\bar{V}_{i, t, N}(l)=0 .
$$

Next, we estimate contribution of small blocks (gaps) $W_{i, t, N}(j), j \geq 1$. Set

$$
\hat{\Gamma}_{i}(l)=\left\{n \in \mathbb{Z}_{+}^{\nu}: \operatorname{dist}\left(n, \cup_{j \leq i} q_{j}(\square(a(l+1)))\right) \leq r(l)\right\},
$$

$\mathcal{G}=\mathcal{F}_{\hat{\Gamma}_{i-1}(l) \cup \hat{\Gamma}_{i}(l-2)}$ and $\mathcal{H}=\mathcal{F}_{U_{r(l)}\left(q_{i}(n)\right)}$ where $n \in \Upsilon(b(l), a(l+1))$. Observe that by the properties of $q_{j}$ 's for any such $n$ and large enough $l$,

$$
\operatorname{dist}\left(\hat{\Gamma}_{i-1}(l) \cup \hat{\Gamma}_{i}(l-2), q_{i}(n)\right) \geq l^{\tau} .
$$

Furthermore, if $j \leq l-2$ then $W_{i, t, N}(j)$ is $\mathcal{F}_{\hat{\Gamma}_{i}(l-2)}$-measurable, and so employing Proposition 3.1 with such $\mathcal{G}$ and $\mathcal{H}$ we obtain similarly to (3.12) relying also on the Cauchy inequality that

$$
\begin{gathered}
\left|E W_{i, t, N}(j) Y_{i, r(l)}\left(q_{i}(n)\right)\right|=\left|E\left(W_{i, t, N}(j) E\left(Y_{i, r(l)}\left(q_{i}(n)\right) \mid \mathcal{G}\right)\right)\right| \\
\leq C_{1}\left\|W_{i, t, N}(j)\right\|_{2} l^{\nu(\tau+\eta+1)} \varpi_{q, p}\left(l^{\tau}-l^{\eta}\right)
\end{gathered}
$$


for some $C_{1}>0$ independent of $t, N, n, l$ and $j \leq l-2$. Hence, by (3.6) and (3.11),

$$
\begin{gathered}
\left|E W_{i, t, N}(j) W_{i, t, N}(l)\right|=\left|E\left(W_{i, t, N}(j) E\left(W_{i, t, N}(l) \mid \mathcal{G}\right)\right)\right| \\
\left.\leq|\Upsilon(b(l), a(l+1))| \max _{n \in \Upsilon(b(l), a(l+1))} \mid E\left(W_{i, t, N}(j) E\left(Y_{i, r(l)}\left(q_{i}(n)\right)\right) \mid \mathcal{G}\right)\right) \mid \\
\left.\leq C_{2}\left\|W_{i, t, N}(j)\right\|_{2} l^{\nu(2 \tau+\eta+2)+2 \eta-\tau-1} \varpi_{q, p}\left(l^{\tau}-l^{\eta}\right)\right)
\end{gathered}
$$

for some $C_{2}>0$ independent of $t, N, n, l$ and $j \leq l-2$. Now we can write

$$
E\left(\sum_{j=0}^{L(N)} W_{i, t, N}(j)\right)^{2} \leq \sum_{j=0}^{L(N)}\left(3 E W_{i, t, N}^{2}(j)\right.
$$

$$
\left.+2 \sum_{l=j-2}^{L(N)}\left|E W_{i, t, N}(j) W_{i, t, N}(l)\right|\right) \leq \sum_{j=0}^{L(N)}\left(3\left\|W_{i, t, N}(j)\right\|_{2}^{2}+C_{3}\left\|W_{i, t, N}(j)\right\|_{2}\right)
$$

where by (2.13), (3.20) and the choice of $\tau$ and $\eta$,

$$
C_{3}=C_{2} \sum_{1 \leq l \leq L(N)} l^{\nu(2 \tau+\eta+2)} \varpi_{p, q}\left(l^{\tau}-l^{\eta}\right) \leq C_{2} \sum_{l=1}^{\infty} l^{5 \nu \tau} \varpi_{p, q}\left(l^{\tau}-l^{\eta}\right)<\infty .
$$

Relying on (2.3), (2.5), (2.15) and the Hölder inequality we can estimate the error of replacement of $Y_{i}\left(q_{i}(n)\right)$ by its $r(l)$-approximation $Y_{i, r(j)}\left(q_{i}(n)\right)$ (see Lemma 3.12 in [17]),

$$
\left\|Y_{i}\left(q_{i}(n)\right)-Y_{i, r(j)}\left(q_{i}(n)\right)\right\|_{2} \leq C_{4} \beta_{q}^{\delta}(r(j))
$$

for some $C_{4}>0$ independent of $i, j$ and $n$. Now, set

$$
\zeta_{i, N}(t)=N^{-\nu / 2} \sum_{1 \leq l \leq L(N)} V_{i, t, N}(l) .
$$

Then by (3.11), (3.21) and (3.22),

$$
\begin{gathered}
\left\|\xi_{i, N}(t)-\zeta_{i, N}(t)\right\|_{2} \leq C_{5} N^{-\nu / 2}\left(\sum_{1 \leq l \leq L(N)} l^{\nu(\tau+1)-1} \beta_{q}^{\delta}\left(\left[l^{\eta}\right]\right)\right. \\
\left.+\left(\sum_{0 \leq l \leq L(N)}\left(\left\|W_{i, t, N}(l)\right\|_{2}^{2}+\left\|W_{i, t, N}(l)\right\|_{2}\right)\right)^{1 / 2}\right) .
\end{gathered}
$$

It follows from (2.15) and Lemma 4.2 of the next section that

$$
\left\|W_{i, t, N}(l)\right\|_{2}^{2}=\mathrm{O}(|\Upsilon(b(l), a(l+1))|) .
$$

By Assumption 2.1 and the choice of $\eta$ and $\tau$ we obtain from (2.14), (3.11), (3.14), (3.23) and (3.24) that

$$
\begin{gathered}
\left\|\xi_{i, N}(t)-\zeta_{i, N}(t)\right\|_{2} \leq C_{6} N^{-\nu / 2}\left(\sum_{1 \leq l \leq L(N)} l^{\nu(\tau+1-5 \eta)-1}\right. \\
\left.+\left(2 \sum_{1 \leq l \leq L(N)} l^{(\nu-1)(\tau+1)+2 \eta}\right)^{1 / 2}\right) \\
\leq C_{7}\left(N^{\nu\left(\frac{1}{2}-\frac{5 \eta}{1+\tau}\right)}+N^{\frac{2 \eta-\tau}{2(1+\tau)}}\right) \rightarrow 0 \text { as } N \rightarrow \infty,
\end{gathered}
$$

and so for each $t$ the limits in distribution as $N \rightarrow \infty$ of $\xi_{i, N}(t)$ and of $\zeta_{i, N}(t)$ coincide (if they exist).

Observe that similarly to (3.7), (3.12) and (3.13) it follows from (2.13), (3.11) together with the contraction property of conditional expectations that

$$
\begin{gathered}
\left|E \zeta_{i, N}(t)\right| \leq N^{-\nu / 2} \sum_{1 \leq l \leq L(N)}\left|E V_{i, t, N}(l)\right| \\
\leq C_{8} N^{-\nu / 2} \sum_{1 \leq l \leq L(N)} l^{\nu(2 \tau+\eta+2)-1} \varpi_{p, q}\left((l-1)^{\tau}-l^{\eta}\right) \\
\leq C_{9} N^{-\nu / 2} \sum_{1 \leq l \leq L(N)} l^{\nu(-3 \tau+\eta+2)-1} \leq C_{10} N^{-\frac{11}{18} \nu},
\end{gathered}
$$

for some $C_{8}, C_{9}, C_{10}>0$ independent of $N$, and so $\left|E \zeta_{i, N}(t)\right| \rightarrow 0$ as $N \rightarrow \infty$. Hence, the Gaussian limiting behavior of $\zeta_{i, N}(t)-E \zeta_{i, N}(t)$ which we will derive 
via mixingale limit theorems yields the same Gaussian limiting behavior of $\zeta_{i, N}(t)$, and so in view of (3.25) the same holds true for $\xi_{i, N}(t)$, as well.

\section{Limiting COVARIANCES}

The first step in our limiting covariances computations is the following estimate of

$$
b_{i, j}(m, n)=E Y_{i}\left(q_{i}(m)\right) Y_{j}\left(q_{j}(n)\right), m, n \in \mathbb{Z}_{+}^{\nu}
$$

where $Y_{i}\left(q_{i}(n)\right)$ was defined in (3.4).

4.1. Lemma. (i) For $i, j=1, \ldots, \ell$ and any $m, n \in \mathbb{Z}_{+}^{\nu}$ set

$$
\begin{aligned}
& \hat{s}_{i, j}(m, n)=\min \left(\min _{1 \leq l \leq j}\left|q_{i}(m)-q_{l}(n)\right|,|m|\right) \\
& \quad \text { and } s_{i, j}(m, n)=\max \left(\hat{s}_{i, j}(m, n), \hat{s}_{j, i}(n, m)\right) .
\end{aligned}
$$

Then for all $i, j \leq k$,

$$
s_{i, j}(m, n) \geq \frac{1}{4 k^{2}}|i m-j n| .
$$

Furthermore, if $i \geq k+1$ then for any $\varepsilon>0$ there exists $M_{\varepsilon}>0$ such that if $\max (|m|,|n|) \geq M_{\varepsilon}$ and $m \neq n$ then

$$
s_{i, i}(m, n) \geq \min \left(|m-n|+\varepsilon^{-1}, \max (|m|,|n|)\right) \geq \frac{1}{2}|m-n| .
$$

(ii) There exists a function $h(l) \geq 0$ defined on integers such that $\sum_{l=1}^{\infty} l^{4 \nu} h(l)<\infty$ and for any $i, j=1,2, \ldots, \ell$ and $l=0,1,2, \ldots$,

$$
\sup _{m, n \in \mathbb{Z}_{+}^{\nu}: s_{i, j}(m, n) \geq l}\left|b_{i, j}(m, n)\right| \leq h(l) .
$$

Proof. (i) Let $i, j \leq k$ and set $u=|i m-j n|$. Then $i|m|+j|n| \geq u$, and so either $|m| \geq \frac{u}{2 i}$ or $|n| \geq \frac{u}{2 j}$. Suppose, for instance, that $|m| \geq \frac{u}{2 i}$. If $\hat{s}_{i, j}(m, n) \geq \frac{u}{4 k^{2}}$ then $s_{i, j}(m, n) \geq \frac{u}{4 k^{2}}$ and we are done. So assume that $s_{i, j}(m, n)<\frac{u}{4 k^{2}}$. Then $\min _{1 \leq l \leq j}|i m-\ln |<\frac{u}{4 k^{2}}$, and so $|i m-\hat{l n}|<\frac{u}{4 k^{2}}$ for some $\hat{l}<j$. Then $i|m|-\hat{l}|n|<$ $\frac{u}{4 k^{2}}$, whence

$$
n>\frac{1}{\hat{l}}\left(i|m|-\frac{u}{4 k^{2}}\right) \geq \frac{u}{2 \hat{l}}-\frac{u}{4 k^{2}} \geq \frac{u}{4 k} .
$$

Next, let $\min _{1 \leq l<i}|j n-l m|=|j n-\tilde{l} m|, \tilde{l}<i$. Then

$$
\begin{gathered}
|j n-\tilde{l} m|=\left|\frac{j}{\hat{l}} \hat{l} n-\frac{j}{\hat{l}} i m+\frac{j}{\hat{l}} i m-\tilde{l} m\right| \geq\left(\frac{j}{\hat{l}} i-\tilde{l}\right)|m|-\frac{j}{\hat{l}}|\hat{l} n-i m| \\
\geq|m|-k \frac{u}{4 k^{2}} \geq \frac{u}{2 i}-\frac{u}{4 k} \geq \frac{u}{4 k} .
\end{gathered}
$$

It follows that $\hat{s}_{j, i}(n, m) \geq \frac{u}{4 k}$, and so by above either $\hat{s}_{i j}(m, n) \geq \frac{u}{4 k^{2}}$ or $\hat{s}_{j, i}(n, m) \geq \frac{u}{4 k}$, whence $s_{i, j}(n, m) \geq \frac{u}{4 k^{2}}$. The case $|n| \geq \frac{u}{2 j}$ is dealt with in the same way exchanging $i$ and $j$ as well as $m$ and $n$ in the above argument.

In order to obtain (4.3) we rely on the definition (4.1) together with the assumptions (2.9) and (2.11).

(ii) By (2.10) there exists $M$ such that $\left|q_{i}(m)-q_{l}(m)\right| \geq|m|$ for all $l<i$ provided $|m| \geq M$. Hence, for such $m$,

$$
\min \left(\min _{1 \leq l \leq j}\left|q_{i}(m)-q_{l}(n)\right|, \min _{1 \leq l<i}\left|q_{i}(m)-q_{l}(m)\right|\right) \geq \hat{s}_{i, j}(m, n) .
$$

Assume that $s_{i, j}(m, n)=\hat{s}_{i, j}(m, n) \geq 2 r$ and set

$$
b_{i, j}^{(r)}(m, n)=E Y_{i, r}\left(q_{i}(m)\right) Y_{j, r}\left(q_{j}(n)\right)
$$


where $Y_{i, r}(l)$ was defined in (3.4). It follows from (2.3), (2.5), (2.6) together with the Hölder inequality (cf. Lemma 3.12 in [17) that

$$
\left|b_{i, j}^{(r)}(m, n)-b_{i, j}(m, n)\right| \leq C\left(\beta_{q}(r)\right)^{\delta}
$$

where a constant $C>0$ does not depend on $i, j, m, n$ and $r$. Set

$$
\Gamma_{r}(m, n)=\cup_{u=1}^{i-1} U_{r}\left(q_{u}(m)\right) \cup_{v=1}^{j} U_{r}\left(q_{v}(n)\right)
$$

where $U_{\rho}(x)=\{y:|x-y| \leq \rho\}$. Applying Proposition 3.1 we conclude that

$$
\begin{gathered}
\left|b_{i, j}^{(r)}(m, n)\right|=\left|E\left(E\left(Y_{i, r}\left(q_{i}(m)\right) \mid \mathcal{F}_{\Gamma_{r}(m, n)}\right) Y_{j, r}\left(q_{j}(n)\right)\right)\right| \\
\leq\left\|Y_{j, r}\left(q_{j}(n)\right)\right\|_{2}\left\|E\left(Y_{i, r}\left(q_{i}(m)\right) \mid \mathcal{F}_{\Gamma_{r}(m, n)}\right)\right\|_{2} \leq C r^{\nu} \varpi_{q, p}\left(s_{i, j}(m, n)-2 r\right) .
\end{gathered}
$$

The estimate in the case $s_{i, j}(m, n)=\hat{s}_{j, i}(n, m) \geq 2 r$ is similar. Now we choose $r=\frac{1}{4} s_{i, j}(m, n)$ and take

$$
h(l)=C\left(l^{\nu} \varpi_{p, q}(2 l)+\left(\beta_{p, q}(l)\right)^{\delta}\right)
$$

provided either $s_{i, j}(m, n)=\hat{s}_{i, j}(m, n)$ and $|m| \geq M$ or $s_{i, j}(m, n)=\hat{s}_{j, i}(n, m)$ and $|n| \geq M$. Next, suppose that $s_{i, j}(m, n)=\hat{s}_{i, j}(m, n)$ and $|m|<M$. Then $\hat{s}_{j, i}(n, m) \leq s_{i, j}(m, n)<M$, and so either $|n|<M$ or $\min _{1 \leq l \leq i}\left|q_{j}(n)-q_{l}(m)\right|<M$. In the latter case we must have $|n| \leq N(M)$ for some $N(M)>0$ depending only on $M$. The same argument holds true when $s_{i, j}(m, n)=\hat{s}_{j, i}(n, m)$ and $|n|<M$. Thus, when $l \geq M$ we can define $h(l)$ by (4.7) while for $l<M$ we can take

$$
h(l)=\max \left\{\left|b_{i, j}(m, n)\right|: 0 \leq|m|,|n| \leq \max (M, N(M)), 1 \leq i, j \leq \ell\right\}
$$

concluding the proof of the lemma.

For $s, t \in[0,1]^{\nu}$ with $s \leq t$ set

$$
\xi_{i, N}(s, t)=N^{-\nu / 2} \sum_{n \in \Delta_{N}(s, t)} F_{i}\left(X\left(q_{1}(n)\right), \ldots, X\left(q_{i}(n)\right)\right) .
$$

Now we can obtain an appropriate estimate of the second moment of $\xi_{i, N}$.

4.2. Lemma. There exists $C>0$ such that for all $t=\left(t_{1}, \ldots, t_{\nu}\right) \geq s=\left(s_{1}, \ldots, s_{\nu}\right) \geq$ 0 and $i=1, \ldots, \ell$,

$$
E\left|\xi_{i, N}(s, t)\right|^{2} \leq C N^{-\nu}\left|\Delta_{N}(s, t)\right| \leq C \prod_{l=1}^{\nu}\left(t_{l}-s_{l}+N^{-1}\right) .
$$

Proof. Set $G(m, l)=\left\{n \in \mathbb{Z}_{+}^{\nu}: l \leq|m-n|<l+1\right\}$. Then by (4.2) and (4.4) for $i \leq k$,

$$
\begin{aligned}
& E\left|\xi_{i, N}(s, t)\right|^{2}=N^{-\nu} \sum_{m, n \in \Delta_{N}(s, t)} b_{i, i}(m, n) \\
\leq & 2 N^{-\nu} \sum_{l=0}^{\infty} \sum_{m \in \Delta_{N}(s, t)} \sum_{n \in G(m, l)}\left|b_{i, i}(m, n)\right| \\
\leq & 2 N^{-\nu} \sum_{l=0}^{\infty} h\left(\left[\frac{l}{4 k^{2}}\right]\right) \sum_{m \in \Delta_{N}(s, t)}|G(m, l)| .
\end{aligned}
$$

If $i>k$ then by (4.3) and (4.4),

$$
\begin{aligned}
& E\left|\xi_{i, N}(s, t)\right|^{2} \leq N^{-\nu} \sum_{m, n:|m|,|n| \leq M_{1}}\left|b_{i, i}(m, n)\right| \\
& +2 N^{-\nu} \sum_{l=0}^{\infty} \sum_{m \in \Delta_{N}(s, t)} \sum_{n \in G(m, l)}\left|b_{i, i}(m, n)\right| \\
& \leq N^{-\nu}\left(C_{1}+\sum_{l=0}^{\infty} h([l / 2]) \sum_{m \in \Delta_{N}(s, t)}|G(m, l)|\right)
\end{aligned}
$$

for some $C_{1}>0$ independent of $s, t$ and $N$. Clearly, for any $l \geq 0$,

$$
|G(m, l)| \leq C_{2}(l+1)^{\nu-1}
$$


for some $C_{2}>0$ independent of $m$ and $l$ since it is bounded by the number of integer points between spheres of radius $l$ and $l+1$. Finally, by (4.9)-(4.11) and the summability of $l^{\nu-1} h(l)$ we derive (4.8).

4.3. Lemma. For each $t>0$ and $i, j \leq k$,

$$
\lim _{u \rightarrow \infty} \limsup _{N \rightarrow \infty} N^{-\nu} \sum_{\substack{0 \leq n, n^{\prime} \leq N t \\\left|i n-j n^{\prime}\right| \geq u}}\left|b_{i, j}\left(n, n^{\prime}\right)\right|=0 .
$$

Proof. Set $G_{i, j}(n, l)=\left\{n^{\prime} \in \mathbb{Z}_{+}^{\nu}: l \leq\left|i n-j n^{\prime}\right|<l+1\right\}$. By Lemma 4.1

$$
\begin{gathered}
N^{-\nu} \sum_{\substack{0 \leq n, n^{\prime} \leq N t \\
\left|i n-j n^{\prime}\right| \geq u}}\left|b_{i, j}\left(n, n^{\prime}\right)\right| \\
\leq N^{-\nu} \sum_{u \leq l<\infty} \sum_{0 \leq n \leq N t} \sum_{n^{\prime} \in G_{i, j}(n, l)}\left|b_{i, j}\left(n, n^{\prime}\right)\right| \\
\leq N^{-\nu} \sum_{u \leq l<\infty} \sum_{0 \leq n \leq N t}\left|G_{i, j}(n, l)\right| h\left(\frac{l}{4 k^{2}}\right) \leq C_{3} \sum_{u \leq l<\infty}(l+1)^{\nu-1} h\left(\frac{l}{4 k^{2}}\right)
\end{gathered}
$$

for some constant $C_{3}>0$ independent of $u$ and $N$. As $u \rightarrow \infty$ the right hand side of (4.12) tends to zero in view of summability of $l^{\nu} h(l)$ in $l$.

4.4. Proposition. For any $i, j \leq k$ and $s=\left(s_{1}, \ldots, s_{\nu}\right), t=\left(t_{1}, \ldots, t_{\nu}\right) \geq 0$ the limit

$$
\lim _{N \rightarrow \infty} N^{-\nu} \sum_{\substack{0 \leq i n \leq N s \\ 0 \leq j n^{\prime} \leq N t, i n-j n^{\prime}=u}} b_{i, j}\left(n, n^{\prime}\right)=\frac{v \prod_{l=1}^{\nu} \min \left(s_{l}, t_{l}\right)}{i j} c_{i, j}(u)
$$

exists for any $u \in \mathbb{Z}^{\nu}$ where $v$ is the greatest common divisor of $i$ and $j, c_{i, j}(u)=0$ if $v$ does not divide all components of $u \in \mathbb{Z}^{\nu}$ and $c_{i, j}(v \tilde{u})$ was defined by (2.23). Finally,

$$
\begin{gathered}
\lim _{N \rightarrow \infty} E \xi_{i, N}(s) \xi_{j, N}(t)=\lim _{N \rightarrow \infty} N^{-\nu} \sum_{\substack{0 \leq i n \leq N s \\
0 \leq j n^{\prime} \leq N t}} b_{i, j}\left(n, n^{\prime}\right) \\
=D_{i, j} \prod_{l=1}^{\nu} \min \left(s_{l}, t_{l}\right)
\end{gathered}
$$

with $D_{i, j}$ defined by (2.22).

Proof. Let $v$ be the greatest common divisor of $i$ and $j$. If $u \in \mathbb{Z}^{\nu}$ has components which are not divisible by $v$ then the sum in (4.13) is empty, and so in this case $c_{i, j}(u)=0$. Thus it remains to deal with this sum when $i n-j n^{\prime}=v u$ for $u \in \mathbb{Z}^{\nu}$. We will show first that the limit

$$
c_{i, j}(u)=\lim _{|n|,\left|n^{\prime}\right| \rightarrow \infty, i n-j n^{\prime}=v u} b_{i, j}\left(n, n^{\prime}\right)
$$

exists. Observe that if we consider two strings $(n, 2 n \ldots, i n)$ and $\left(n^{\prime}, 2 n^{\prime}, \ldots, j n^{\prime}\right)$ with $i n-j n^{\prime}=v u$ then there will also be pairs $\left(i_{\alpha}, j_{\alpha}\right), \alpha=1,2, \ldots, v-1$ such that $i_{\alpha} n-j_{\alpha} n^{\prime}=\alpha u$ where $i_{\alpha}=\alpha i_{1}$ and $j_{\alpha}=\alpha j_{1}$ with $i_{1}$ and $j_{1}$ being coprime. On the other hand, if $\tilde{i} / \tilde{j} \neq i_{1} / j_{1}$ then

$$
\begin{gathered}
\left|\tilde{i} n-\tilde{j} n^{\prime}\right|=\tilde{j}\left|\tilde{i} \frac{\tilde{i}}{\tilde{j}} n-n^{\prime}\right|=\tilde{j}\left|\left(\frac{\tilde{i}}{\tilde{j}}-\frac{i_{1}}{j_{1}}\right) n+\frac{1}{j_{1}}\left(i_{1} n-j_{1} n^{\prime}\right)\right| \\
\geq|n| \tilde{j}\left|\frac{\tilde{i}}{\tilde{j}}-\frac{i_{1}}{j_{1}}\right|-\frac{\tilde{j}}{j_{1}}|u| \rightarrow \infty \text { as }|n| \rightarrow \infty .
\end{gathered}
$$

We split the collection of numbers $\left(n, 2 n, \ldots, i n ; n^{\prime}, 2 n^{\prime}, \ldots, j n^{\prime}\right)$ into disjoint sets $\Gamma_{1}, \ldots, \Gamma_{v}, \ldots, \Gamma_{i+j-v}$ where $\Gamma_{\alpha}=\left\{\alpha i_{1}, \alpha j_{1}\right\}, \alpha=1, \ldots, v$ are pairs and $\Gamma_{v+\beta}, \beta=$ $1,2, \ldots, i+j-v$ are singeltons. We order the latter so that $\Gamma_{\beta}=\left\{l_{\beta} n\right\}, 1 \leq$ $l_{\beta}<i, \beta=v+1, \ldots, i$ with $l_{\beta} \neq \alpha i_{1}$ for $\alpha=1, \ldots, v$ and $\Gamma_{v+\beta}=\left\{l^{\prime}{ }_{\beta} n^{\prime}\right\}$ for 
$\beta=i+1, \ldots, i+j-v, 1 \leq l^{\prime}{ }_{\beta}<i, l^{\prime}{ }_{\beta} \neq \alpha j_{1}$ for $\alpha=1, \ldots, v$. By (4.16) there is $\delta>0$ depending on $u$ but not on $n$ and $n^{\prime}$ such that

$$
\min _{1 \leq l \neq l^{\prime} \leq i+j-v} \operatorname{dist}\left(\Gamma_{l}, \Gamma_{l^{\prime}}\right) \geq \delta n .
$$

Set

$$
U_{r}\left(\Gamma_{l}\right)=\left\{n \in \mathbb{Z}_{+}^{\nu}: \operatorname{dist}\left(n, \Gamma_{l}\right) \leq r\right\}, l=1,2, \ldots, i+j-v
$$

and choose $r=r(n) \rightarrow \infty$ as $|n| \rightarrow \infty$ so that all $U_{r(n)}\left(\Gamma_{l}\right), l=1,2, \ldots, i+j-v$ were disjoint.

Now, observe that $b_{i, j}\left(n, n^{\prime}\right)$ has the form $E G\left(Y_{1}\left(n, n^{\prime}\right), Y_{2}\left(n, n^{\prime}\right), \ldots, Y_{i+j-v}\left(n, n^{\prime}\right)\right)$ where $Y_{\alpha}\left(n, n^{\prime}\right)=\left(X(\alpha i, n), X\left(\alpha j, n^{\prime}\right)\right)$ for $\alpha=1, \ldots, v, Y_{\beta}\left(n, n^{\prime}\right)=X\left(l_{\beta} n\right)$ for $\beta=v+1, \ldots, i$ and $Y_{\beta}\left(n, n^{\prime}\right)=X\left(l_{\beta}^{\prime} n^{\prime}\right)$ for $\beta=i+1, \ldots, i+j-v$. Define $G_{1}=G$ and successively

$$
G_{l+1}\left(y_{l+1}, y_{l+2}, \ldots, y_{i+j-v}\right)=E G\left(Y_{l}\left(n, n^{\prime}\right), y_{l+1}, y_{l+2}, \ldots, y_{i+j-v}\right) .
$$

Relying on the assumptions (2.13) and (2.14) we can apply (3.3) of Proposition 3.1 with $V=\left(Y_{l+1}\left(n, n^{\prime}\right), Y_{l+2}\left(n, n^{\prime}\right), \ldots, Y_{i+j-v}\left(n, n^{\prime}\right)\right), Z=Y_{l}\left(n, n^{\prime}\right), \mathcal{G}=\mathcal{F}_{\tilde{U}}$ with $\tilde{U}=\cup_{l+1 \leq \beta \leq i+j-v} U_{r(n)}\left(\Gamma_{\beta}\right)$ and $\mathcal{H}=\mathcal{F}_{U_{l}(r(n))}$ obtaining that

$$
\begin{gathered}
E G_{l}\left(Y_{l}\left(n, n^{\prime}\right), Y_{l+1}\left(n, n^{\prime}\right), \ldots, Y_{i+j-v}\left(n, n^{\prime}\right)\right) \\
-E G_{l+1}\left(Y_{l+1}\left(Y_{l+1}\left(n, n^{\prime}\right), \ldots, Y_{i+j-v}\left(n, n^{\prime}\right)\right) \rightarrow 0 \text { as } n \rightarrow \infty .\right.
\end{gathered}
$$

This argument repeated for $l=1,2, \ldots, i+j-v-1$ yields (4.15) with $c_{i, j}$ given by (2.23).

Finally, in order to obtain (4.13) and (4.14) we have to count the number of solutions $n, n^{\prime}$ of the vector Diophantine equation $i n-j n^{\prime}=v u$ where $0 \leq i n \leq N s$ and $0 \leq j n^{\prime} \leq N t$. Since we have to satisfy this equation coordinate wise, the number of solutions is the product of the number of solutions in each coordinate. Let, as before, $i=v i_{1}$ and $j=v j_{1}$ with $i_{1}$ and $j_{1}$ being coprime then all solutions of the equation $i_{1} n_{l}-j_{1} n^{\prime}{ }_{l}=u_{l}$ are given by $n=n_{0}+j_{1} m$ and $n^{\prime}=n_{0}^{\prime}+i_{1} m$ where $n_{0}, n_{0}^{\prime}$ is its particular solution and $m$ is any integer. The number of such solutions with $0 \leq i n_{l} \leq N s_{l}$ and $0 \leq j n_{l}^{\prime} \leq N t_{l}$ for large $N$ is equal approximately to

$$
N \min \left(\frac{t_{l}}{i j_{1}}, \frac{s_{l}}{j i_{1}}\right)+O(1)=\frac{N v \min \left(s_{l}, t_{l}\right)}{i j}+O(1)
$$

and taking the product in $l$ we obtain (4.13) while (4.14) follows from (4.13) and Lemmas 4.1 and 4.3 .

4.5. Proposition. For $i \geq k+1$,

$$
\lim _{N \rightarrow \infty} E\left|\xi_{i, N}(t)\right|^{2}=\left(\prod_{l=1}^{\nu} t_{l}\right) \int\left(F_{i}\left(x_{1}, x_{2}, \ldots, x_{i}\right)\right)^{2} d \mu\left(x_{1}\right) d \mu\left(x_{2}\right) \cdots d \mu\left(x_{i}\right) .
$$

Moreover, for any $t, s \in \mathbb{R}_{+}^{\nu}$ and $j<i$,

$$
\lim _{N \rightarrow \infty} E\left(\xi_{i, N}(t) \xi_{j, N}(s)\right)=0 .
$$

Proof. By (2.9)-(2.11), (4.3) and (4.4),

$$
b_{i, i}(m, n) \rightarrow 0 \text { as } \max (|m|,|n|) \rightarrow \infty \text { with }|m-n| \geq 1 .
$$


Therefore, by (4.3) and (4.4) for any fixed $L$,

$$
\begin{aligned}
& \lim \sup _{N \rightarrow \infty} N^{-\nu} \sum_{m, n \in \Delta_{N}(t), m \neq n}\left|b_{i, i}(m, n)\right| \\
& \leq \lim \sup _{N \rightarrow \infty} N^{-\nu}\left(\sum_{1 \leq|m-n| \leq L}\left|b_{i, i}(m, n)\right|\right. \\
& \left.+C\left|\Delta_{N}(t)\right| \sum_{l \geq L} l^{\nu-1} h(l)\right)=\tilde{C} \sum_{l \geq L / 2} l^{\nu-1} h(l)
\end{aligned}
$$

for some $C, \tilde{C}>0$ independent of $N$ and $L$. We now let $L \rightarrow \infty$ and since $l^{\nu-1} h(l)$ is summable it follows that lim sup in the left hand side above equals zero, i.e. the off-diagonal terms do not contribute in (4.17). It remains to deal with the diagonal terms $b_{i, i}(n, n)$. Since $\left|q_{j}(n)-q_{j-1}(n)\right| \rightarrow \infty$ for $j=2,3, \ldots, \ell$ as $|n| \rightarrow \infty$ it follows by the argument similar to one applied in the proof of Proposition 4.4 (see a more general Lemma 4.3 in [17]) that

$$
\lim _{|n| \rightarrow \infty} b_{i, i}(n, n)=\int\left(F_{i}\left(x_{1}, x_{2}, \ldots, x_{i}\right)\right)^{2} d \mu\left(x_{1}\right) \cdots d \mu\left(x_{i}\right) .
$$

Namely, set $G_{i}\left(x_{1}, \ldots, x_{i}\right)=\left(F_{i}\left(x_{1}, x_{2}, \ldots, x_{i}\right)\right)^{2}$ and recursively for $l=i-$ $1, \ldots, 2,1,0$,

$$
G_{l}\left(x_{1}, \ldots, x_{l}\right)=\int G_{l+1}\left(x_{1}, \ldots, x_{l+1}\right) d \mu\left(x_{l+1}\right) .
$$

Taking into account that $\left|q_{l}(n)-q_{\tilde{l}}(n)\right| \rightarrow \infty$ as $|n| \rightarrow \infty$ when $l \neq \tilde{l}$ we apply Proposition 3.1 to obtain successively for $l=i, i-1, \ldots, 1,0$ that

$\left|E G_{l+1}\left(X\left(q_{1}(n)\right), \ldots, X\left(q_{l}(n)\right)\right)-E G_{l+1}\left(X\left(q_{1}(n)\right), \ldots, X\left(q_{l}(n)\right)\right)\right| \rightarrow 0$ as $|n| \rightarrow \infty$.

Since $b_{i, i}(n, n)=G_{0}$ we arrive at (4.19).

Next, we deal with (4.18). Since $i>j$ and $i>k$ then by (2.9)-(2.11) for any $\varepsilon>0$ there exists $N(\varepsilon)$ such that whenever $|m|>\varepsilon N$ and $|n| \leq N \sqrt{\nu}$ we have $s_{i, j}(m, n) \geq \min \left(|m-n|+\varepsilon^{-1},|m|\right)$ provided $N \geq N(\varepsilon)$ where $s_{i, j}(m, n)$ is the same as in Lemma 4.1. It follows from Lemmas 4.1 and 4.2 that

$$
\begin{gathered}
\left|E \xi_{i, N}(t) \xi_{j, N}(s)\right| \leq\left|\sum_{|m| \leq \varepsilon N, n \in \Delta_{N}(s)} b_{i, j}(m, n)\right| \\
+N^{-\nu} \sum_{|m|>\varepsilon N, m \in \Delta_{N}(t), n \in \Delta_{N}(s)}\left|b_{i, j}(m, n)\right| \\
\leq N^{-\nu}\left(\sum_{|m| \leq \varepsilon N} E Y_{i, m}^{2}\right)^{1 / 2}\left(\sum_{n \in \Delta_{N}(s)} E Y_{j, n}^{2}\right)^{1 / 2} \\
+C \sum_{l \geq \min \left(\varepsilon^{-1}, \varepsilon N\right)} l^{\nu-1} h(l) \leq C \sqrt{\varepsilon}+C \sum_{l \geq \min \left(\varepsilon^{-1}, \varepsilon N\right)} l^{\nu-1} h(l)
\end{gathered}
$$

for some $C>0$ independent of $N$ and $\varepsilon$. Letting in (4.20), first $N \rightarrow \infty$ and then $\varepsilon \rightarrow 0$ we arrive at (4.18).

\section{Tightness estimates}

First, we will extend the estimate of Lemma 4.2 to the corresponding estimate of the 4th moment.

5.1. Lemma. There exists $C>0$ such that for all $t=\left(t_{1}, \ldots, t_{\nu}\right) \geq s=\left(s_{1}, \ldots, s_{\nu}\right) \geq$ 0 and $i=1, \ldots, \ell$,

$$
E\left|\xi_{i, N}(s, t)\right|^{4} \leq C N^{-2 \nu}\left|\Delta_{N}(s, t)\right|^{2} \leq C \prod_{l=1}^{\nu}\left(t_{l}-s_{l}+N^{-1}\right)^{2} .
$$


Proof. For $n^{(1)}, n^{(2)}, n^{(3)}, n^{(4)} \in \mathbb{Z}_{+}^{\nu}$ set

$$
d_{i}\left(n^{(1)}, n^{(2)}, n^{(3)}, n^{(4)}\right)=E Y_{i, q_{i}\left(n^{(1)}\right)} Y_{i, q_{i}\left(n^{(2)}\right)} Y_{i, q_{i}\left(n^{(3)}\right)} Y_{i, q_{i}\left(n^{(4)}\right)}
$$

and for $r>0$,

$$
d_{i}^{(r)}\left(n^{(1)}, n^{(2)}, n^{(3)}, n^{(4)}\right)=E Y_{i, q_{i}\left(n^{(1)}\right), r} Y_{i, q_{i}\left(n^{(2)}\right), r} Y_{i, q_{i}\left(n^{(3)}\right), r} Y_{i, q_{i}\left(n^{(4)}\right), r} .
$$

Then similarly to (4.5),

$$
\left|d_{i}^{(r)}\left(n^{(1)}, n^{(2)}, n^{(3)}, n^{(4)}\right)-d_{i}\left(n^{(1)}, n^{(2)}, n^{(3)}, n^{(4)}\right)\right| \leq C_{1}\left(\beta_{q}(r)\right)^{\delta}
$$

where $C_{1}>0$ does not depend on $n^{(1)}, n^{(2)}, n^{(3)}, n^{(4)}$ and $r$. Define

$$
\begin{gathered}
v_{i}\left(n^{(1)}, n^{(2)}, n^{(3)}, n^{(4)}\right)=\max _{1 \leq j \leq 4}\left(\operatorname { m i n } \left(\min _{l<i}\left|q_{i}\left(n^{(j)}\right)-q_{l}\left(n^{(j)}\right)\right|,\right.\right. \\
\left.\left.\min _{\tilde{j} \neq j, l \leq i}\left|q_{i}\left(n^{(j)}\right)-q_{l}\left(n^{(\tilde{j})}\right)\right|\right)\right) .
\end{gathered}
$$

Without loss of generality assume that

$$
v_{i}\left(n^{(1)}, n^{(2)}, n^{(3)}, n^{(4)}\right)=\min \left(\min _{l<i}\left|q_{i}\left(n^{(j)}\right)-q_{l}\left(n^{(j)}\right)\right|, \min _{\tilde{j} \neq j, l \leq i}\left|q_{i}\left(n^{(j)}\right)-q_{l}\left(n^{(\tilde{j})}\right)\right|\right) .
$$

For each $a \geq 0$ introduce the sets

$$
\Gamma_{a}=\left\{n^{(1)}, n^{(2)}, n^{(3)}, n^{(4)} \in \mathbb{Z}_{+}: a \leq v_{i}\left(n^{(1)}, n^{(2)}, n^{(3)}, n^{(4)}\right)<a+1\right\}
$$

and

$$
\Gamma_{a}(N, s, t)=\left\{\left(n^{(1)}, n^{(2)}, n^{(3)}, n^{(4)}\right) \in \Gamma_{a}: n^{(1)}, n^{(2)}, n^{(3)}, n^{(4)} \in \Delta_{N}(s, t)\right\} .
$$

If $i \leq k$ and $\left(n^{(1)}, n^{(2)}, n^{(3)}, n^{(4)}\right) \in \Gamma_{a}$ then for $j=2,3,4$,

either $\left.\mid n^{(j)}\right)<a+1$ or $\left|i n^{(j)}-\ln ^{(\tilde{j})}\right|<a+1$ for some $l=1, \ldots, i$ and $\tilde{j} \neq j$.

It follows that

$$
\left|\Gamma_{a}(N, s, t)\right| \leq C_{2} a^{2}\left(1+a^{2}+\left|\Delta_{N}(s, t)\right|^{2}\right)
$$

for some $C_{2}>0$ independent of $a, N, s$ and $t$. If $i \geq k+1$ then by (2.9)-(2.11) there exists $M>0$ such that whenever $|n| \geq M$,

$$
\min _{l<i}\left|q_{i}(n)-q_{l}(n)\right| \geq|n| \text { and } \min _{\tilde{i} \leq i, \tilde{n} \neq n}\left|q_{i}(n)-q_{\tilde{i}}(\tilde{n})\right| \geq|n-\tilde{n}| .
$$

Then similarly to the case $i \leq k$ we conclude from (5.5) that (5.4) holds true also when $i \geq k+1$.

Next, let $r=a / 3$ and $\left(n^{(1)}, n^{(2)}, n^{(3)}, n^{(4)}\right) \in \Gamma_{a}$ satisfy (5.3). Set

$$
\Psi_{r}\left(n^{(1)}, n^{(2)}, n^{(3)}, n^{(4)}\right)=\cup_{u=1}^{i-1} U_{r}\left(q_{u}\left(n^{(1)}\right)\right) \cup\left(\cup_{j=2}^{4} \cup_{v=1}^{i} U_{r}\left(q_{v}\left(n^{(j)}\right)\right) .\right.
$$

Then by Proposition 3.1 similarly to (4.6) we derive that

$$
\begin{aligned}
& \left|d_{i}^{(r)}\left(n^{(1)}, n^{(2)}, n^{(3)}, n^{(4)}\right)\right| \leq \mid E\left(E\left(Y_{i, q_{i}\left(n^{(1)}\right), r} \mid \mathcal{F}_{\Psi_{r}\left(n^{(1)}, n^{(2)}, n^{(3)}, n^{(4)}\right)}\right)\right. \\
& \left.\quad \times Y_{i, q_{i}\left(n^{(2)}\right), r} Y_{i, q_{i}\left(n^{(3)}\right), r} Y_{i, q_{i}\left(n^{(4)}\right), r}\right) \mid \leq C_{3} a^{\nu} \varpi_{q, p}(a / 3)
\end{aligned}
$$

for some $C_{3}>0$ independent of $a, n^{(1)}, n^{(2)}, n^{(3)}$ and $n^{(4)}$. Set $q(a)=$ $C_{1}\left(\beta_{q}(a / 3)\right)^{\delta}+C_{3} a^{\nu} \varpi_{q, p}(a / 3)$. Then

$$
\begin{aligned}
(5.7) E\left|\xi_{i, N}(s, t)\right|^{4} \leq & \sum_{a=0}^{\infty} \sum_{\left(n^{(1)}, n^{(2)}, n^{(3)}, n^{(4)}\right) \in \Gamma_{a}(N, s, t)}\left|d_{i}^{(r)}\left(n^{(1)}, n^{(2)}, n^{(3)}, n^{(4)}\right)\right| \\
& \leq C_{2} \sum_{a=0}^{\infty} q(a) a^{2}\left(1+a^{2}+\left|\Delta_{N}(s, t)\right|^{2}\right)
\end{aligned}
$$

and (5.1) follows from (5.7) and Assumption 2.1. 
Now tightness of each sequence of random fields $\left\{\xi_{i, N}(t), t \in[0,1]^{\nu}\right\}$ follows by a slight modification of [9] (see also Ch.5 in [8] and Theorem 1.4.7 in [16]), and so the sequence of random fields $\left\{\xi_{N}(t), t \in[0,1]^{\nu}\right\}$ is tight, as well.

\section{Gaussian Limits}

For each fixed $t \in[0,1]^{\nu}$ the convergence in distribution as $N \rightarrow \infty$ of each $\zeta_{i, N}(t), i=1, \ldots, \ell$ to corresponding Gaussian (maybe degenerated) random variables follows from central limit theorems for mixingale arrays (see 20, 14 and references there) in view of the mixingale estimates of Section 3, convergence of covariances obtained in Section 4 and the tightness result derived in Section 5 , Then $\xi_{i, N}(t), i=1, \ldots, \ell$ also converge in distribution to the same Gaussian random variables in view of (3.25). Furthermore, for any $\mathbf{t}=\left(t^{(1)}, \ldots, t^{(j)}\right), t^{(a)} \in[0,1]^{\nu}, a=$ $1, \ldots, j$ and $\mathbf{d}=\left(d_{1}, \ldots, d_{j}\right)$ set

$$
V_{i, \mathbf{t}, \mathbf{d}, N}(l)=\sum_{a=1}^{j} d_{a} V_{i, t^{(a)}, N}(l)
$$

and

$$
\zeta_{i, \mathbf{d}, N}(\mathbf{t})=\sum_{a=1}^{j} d_{a} \zeta_{i, N}\left(t^{(a)}\right)=N^{-\nu / 2} \sum_{1 \leq l \leq L(N)} V_{i, \mathbf{t}, \mathbf{d}, N}(l) .
$$

Then, we obtain from (3.13) and (3.18) similar mixingale estimates also for $V_{i, \mathbf{t}, \mathbf{d}, N}(l)$ which via [20] and [14] yields convergence in distribution as $N \rightarrow \infty$ to Gaussian random variables of each $\zeta_{i, \mathbf{d}, N}(\mathbf{t})$. This together with (3.25) imply that each

$$
\xi_{i, \mathbf{d}, N}(\mathbf{t})=\sum_{a=1}^{j} d_{a} \xi_{i, N}\left(t^{(a)}\right)
$$

converges in distribution as $N \rightarrow \infty$ to Gaussian random variables. Hence, finite dimensional distributions of each $\xi_{i, N}$ have Gaussian limits which together with tightness results of Section 5 yields that each $\xi_{i, N}$ converges in distribution as $N \rightarrow \infty$ to a Gaussian random field $\eta_{i}$.

In fact, we can show that $\left(\xi_{1, N}, \ldots, \xi_{1, k}\right)$ converges in distribution as $N \rightarrow \infty$ to a $k$-dimensional Gaussian random field $\left(\eta_{1}, \ldots, \eta_{k}\right)$. Indeed, for any $\mathbf{e}=\left(e_{1}, \ldots, e_{k}\right) \in$ $\mathbb{R}^{k}$ set

$$
V_{\mathbf{t}, \mathbf{d}, \mathbf{e}, N}(l)=\sum_{i=1}^{k} e_{i} V_{i, \mathbf{t}, \mathbf{d}, N}(l) \text { and } \zeta_{\mathbf{d}, \mathbf{e}, N}(\mathbf{t})=\sum_{i=1}^{k} e_{i} \zeta_{i, \mathbf{d}, N}(\mathbf{t}) .
$$

Then it is easy to see again by (3.13) and (3.18) that similar mixingale estimates hold true also for $V_{\mathbf{t}, \mathbf{d}, \mathbf{e}, N}(l)$ which via [20] and 14 provides convergence in distribution as $N \rightarrow \infty$ of $\zeta_{\mathbf{d}, \mathbf{e}, N}$ to a Gaussian random variable which must have the same distribution as $\sum_{i=1}^{k} \sum_{a=1}^{j} e_{i} \eta_{i}\left(t^{(a)}\right)$. As above we conclude from (3.25) and tightness arguments of Section 5 that, in fact, $\sum_{i=1}^{k} e_{i} \xi_{i, N}$ converges in distribution as $N \rightarrow \infty$ to a Gaussian random field which must have the same distribution as $\sum_{i=1}^{k} e_{i} \eta_{i}$. Thus, $\left(\eta_{1}, \ldots, \eta_{k}\right)$ is a Gaussian random field and $\left(\xi_{1, N}, \ldots, \xi_{k, N}\right)$ converges in distribution to it as $N \rightarrow \infty$. Finally, $\sum_{i=1}^{k} \xi_{i, N}(i t)$ converges in distribution as $N \rightarrow \infty$ to the random field $\sum_{i=1}^{k} \eta_{i}(i t)$ which must be Gaussian as a 
result of the linear transformation (in the path space) of a Gaussian random field (see, for instance, [4, Section 2.2).

Next, clearly, $\xi_{N}$ converges in distribution to $\xi$ given by (2.24) and it remains to show that $\eta_{i}$ with $i \geq k+1$ are independent of each other and of $\eta_{i}$ with $i \leq k$ which will imply that $\xi$ is a Gaussian random field. This can be done either via a modified version of Theorem 5.6 from [17] or by the following more direct approach. First, observe that (2.11) implies that there exists $\varepsilon_{N} \rightarrow 0$ as $N \rightarrow \infty$ such that

$$
\lim _{N \rightarrow \infty} \min _{n, \tilde{n} \in \Delta_{N}(\mathbf{1}) \backslash \Delta_{N}\left(\varepsilon_{N} \mathbf{1}\right)}\left(\left|q_{i}(\tilde{n})\right|-\max _{l<i}\left|q_{l}(n)\right|-|\tilde{n}|\right)=\infty
$$

where $\mathbf{1}=(1, \ldots, 1) \in[0,1]^{\nu}$. We see from (6.5) that if $n, \tilde{n} \in \Delta\left(\varepsilon_{n} \mathbf{1}, \mathbf{t}\right)$ then any $q_{i}(n), i \geq k+1$ and $q_{j}(\tilde{n}), j \neq i$ are widely separated. Next, it follows from Lemma 4.2 that for each $i$,

$$
\xi_{i, N}\left(\varepsilon_{N} \mathbf{1}\right) \rightarrow 0 \text { in probability as } N \rightarrow \infty,
$$

and so in all our arguments the sum over $\Delta_{N}\left(\varepsilon_{N} \mathbf{1}\right)$ can be disregarded.

In order to use (6.5) and (6.6) introduce $j_{N}=\min \left\{j: a(j) \geq \varepsilon_{N} N\right\}$ with $a(j)$ defined in (3.5). For $\mathbf{t}=\left(t^{(1)}, \ldots, t^{(j)}\right), t^{(a)} \in\left[\varepsilon_{N}, 1\right]^{\nu}, a=1, \ldots, j, \mathbf{d}=\left(d_{1}, \ldots, d_{j}\right)$ and $\mathbf{e}=\left(e_{k+1}, \ldots, e_{\ell}\right)$ define for $l=1,2, \ldots, L(N)$,

$$
\tilde{V}_{\mathbf{t}, \mathbf{d}, \mathbf{e}, N}(l)=\sum_{a=1}^{j} \sum_{i=k+1}^{\ell} d_{a} e_{i} V_{i, t^{(a)}, N}(l)
$$

as in (6.1) and (6.4). On the other hand, for $(i-k) L(N)+j_{N} \leq l \leq(i+1-k) L(N)$ and $i \geq k+1$ we set

$$
\tilde{V}_{\mathbf{t}, \mathbf{d}, \mathbf{e}, N}(l)=\sum_{a=1}^{j} d_{a} e_{i} V_{i, t^{(a)}, N}(l-(i-k) L(N)
$$

setting $\tilde{V}_{\mathbf{t}, \mathbf{d}, \mathbf{e}, N}(l)$ to be zero if it is not defined by one of the above and assuming that $t^{(a)}>\varepsilon_{N}$ for all $a$. It is easy to see from (2.9) (2.11), (6.5), (6.6) and mixingale estimates of Section 3 that the sequence $\left(\tilde{V}_{\mathbf{t}, \mathbf{d}, \mathbf{e}, N}(l)-E \tilde{V}_{\mathbf{t}, \mathbf{d}, \mathbf{e}, N}(l)\right) N^{-\nu / 2}, l=$ $j_{N}, \ldots, \ell L(N)$ forms a mixingale array with the corresponding $\sigma$-algebras $\mathcal{G}_{l}=\mathcal{G}_{l}^{(i)}$ for $i \leq k, \mathcal{G}_{l+(i-k) L(N)}=\mathcal{G}_{l}^{(i)}$ for $i \geq k+1$ and estimates similar to (3.17). Observe that

$$
\tilde{\zeta}_{\mathbf{d}, \mathbf{e}, N}(\mathbf{t})=N^{\nu / 2} \sum_{j_{N} \leq l \leq L(N)} \sum_{i=1}^{\ell} e_{i} V_{i, \mathbf{t}, \mathbf{d}, N}(l)=N^{-\nu / 2} \sum_{1 \leq l \leq \ell L(N)} \tilde{V}_{\mathbf{t}, \mathbf{d}, \mathbf{e}, N}(l) .
$$

Taking into account (6.6), mixingale estimates of Section 3 and convergence of covariances results of Section 4 and tightness arguments of Section 5 we conclude by mixingale limit theorems from [20] and [14] that $\tilde{\zeta}_{\mathbf{d}, \mathbf{e}, N}(\mathbf{t})-E \tilde{\zeta}_{\mathbf{d}, \mathbf{e}, N}(\mathbf{t})$ converges in distribution as $N \rightarrow \infty$ to a Gaussian random variable while similarly to the end of Section 3 we see that $\tilde{\zeta}_{\mathbf{d}, \mathbf{e}, N}(\mathbf{t})$ converges to the same limit. It follows from here in view of (3.25) and (6.6) that each linear combination $\sum_{a=1}^{j} \sum_{i=1}^{\ell} d_{a} e_{i} \xi_{i, N}\left(t^{(a)}\right)$ converges in distribution as $N \rightarrow \infty$ to some Gaussian random variable which then must be $\sum_{a=1}^{j} \sum_{i=1}^{\ell} d_{a} e_{i} \eta_{i}\left(t^{(a)}\right)$. Thus $\left(\eta_{1}, \eta_{2}, \ldots, \eta_{\ell}\right)$ is an $\ell$-dimensional Gaussian random field. Invoking again the linear transformation argument from Section 2.2 of 4 we conclude that the field $\xi(t)$ given by (2.24) is a Gaussian one and taking into account the vanishing limiting covariances result (4.18) we obtain also independency of $\eta_{i}, i \geq k+1$ of each other and of $\eta_{i}$ 's with $i \leq k$. 


\section{REFERENCES}

[1] K.S. Alexander, Mixing properties and exponential decay for lattice systems in finite volumes, Ann. Probab. 32 (2004), 441-487.

[2] T. Austin, On the norm convergence of non-conventional ergodic averages, Ergod. Th.\& Dynam. Sys. 30 (2010), 321-338.

[3] V. Bergelson, Weakly mixing PET, Ergod. Th.\& Dynam. Sys. 7 (1987), 337-349 .

[4] V.I. Bogachev, Gaussian measures, Amer. Math. Soc., Providence, 1998.

[5] E. Bolthausen, On the central limit theorem for stationary mixing random fields, Ann. Probab. 10 (1982), 1047-1050.

[6] R.C. Bradley, Introduction to Strong Mixing Conditions, Kendrick Press, Heber City, 2007.

[7] A. Bulinski and A. Shashkin, Strong invariance principle for dependent random fields, IMS Lecture Notes, Dynamics\& Stochastics 48 (2006), 128-143.

[8] A. Bulinski and A. Shashkin, Limit Theorems for Associated Random Fields and Related Systems, World Scientific, Singapore, 2007.

[9] P.J. Bickel and M.J. Wichura, Convergence criteria for multiparameter stochastic processes and some applications, Ann. Math. Stat. 42 (1971), 1656-1670.

[10] R.L. Dobruschin, The description of a random field by means of conditional probabilities and conditions of its regularity, Th. Probab. Appl. 13 (1968), 197-224.

[11] N. Dunford and J.T. Schwartz, Linear Operators, Part I, Wiley, New York, 1958.

[12] H. Furstenberg, Nonconventional ergodic averages, Proc. Symp. Pure Math. 50, 43-56 (1990).

[13] D.J.H. Garling, Inequalities: a Journey into Linear Analysis, Cambridge Univ. Press, Cambridge (2007).

[14] R.M. de Jong, Limit theorems for dependent heterogeneous random variables, Econometric Th. 13, 353-367 (1997).

[15] Yu. Kifer, Nonconventional limit theorems, Probab. Th. Rel. Fields, 148, 71-106 (2010).

[16] H. Kunita, Stochastic Flows and Stochastic Differential Equations, Cambridge Univ. Press, Cambridge (1990).

[17] Yu. Kifer and S.R.S. Varadhan, Nonconventional limit theorems in discrete and continuous time via martingales, Preprint, arXiv: 1012.2223.

[18] A. Leibman, Convergence of multiple ergodic averages along polynomials of several variables, Israel J. Math. 146 (2005), 303-315.

[19] D.L. McLeish, Invariance principles for dependent variables, Z. Wahrsch. verw. Geb. 32 (1975), 165-178.

[20] D.L. McLeish, On the invariance principle for nonstationary mixingales, Ann. Probab. 5 (1977), 616-621.

[21] J. Sunklodas, On normal approximation for strongly mixing random fields, Th. Probab. Appl. 52 (2008), 125-132.

E-mail address: kifer@math.huji.ac.il

Institute of Mathematics, Hebrew University, Jerusalem 91904, ISRAEL 